\title{
The efficacy of mobile application use on recall of surgical risks in nasal bone fracture reduction surgery
}

\author{
Choong Hyeon Kim, \\ Ji Seon Cheon, \\ Woo Young Choi, \\ Kyung Min Son \\ Department of Plastic and Reconstructive \\ Surgery, Chosun University College of \\ Medicine, Gwangju, Korea
}

This article contains Supplemental Program File S1.

\begin{abstract}
Background: The number of surgical risks recalled by a patient after surgery can be used as a parameter for assessing how well the patient has understood the informed consent process. No study has investigated the usefulness of a self-developed mobile application in the traditional informed consent process in patients with a nasal bone fracture. This study aimed to investigate whether delivery of information, such as surgical risks, through a mobile application is more effective than delivery of information through only verbal means and a paper.

Methods: This prospective, randomized study included 60 patients with a nasal bone fracture. The experimental group $(n=30)$ received preoperative explanation with the traditional informed consent process in addition to a mobile application, while the control group $(n=30)$ received preoperative explanation with only the traditional informed consent process. Four weeks after surgery, the number of recalled surgical risks was compared for analysis. The following six surgical risks were explained: pain, bleeding, nasal deformity, numbness, nasal obstruction, and nasal cartilage necrosis.

Results: The mean number of recalled surgical risks among all patients was $1.58 \pm 0.56$. The most frequently recalled surgical risk was nasal deformity in both groups. The mean number of recalled surgical risks was $1.72 \pm 0.52$ in the experimental group and $1.49 \pm 0.57$ in the control group. There was a significant association between mobile application use and the mean number of recalled surgical risks $(p=0.047)$. Age, sex, and the level of education were not significantly associated with the mean number of recalled surgical risks.

Conclusion: This study found that a mobile application could contribute to the efficient delivery of information during the informed consent process. With further improvement, it could be used in other plastic surgeries and other surgeries, and such an application can potentially be used for explaining risks as well as delivering other types of information.
\end{abstract}

Keywords: Mobile applications / Informed consent / Fractures

\section{INTRODUCTION}

Informed consent involves providing information about the condition, purpose, and risks of treatment, as well as the possible outcomes [1]. Particularly, explanation about surgical risks is an important aspect of the informed consent process [1]. Apart from the

Correspondence: Ji Seon Cheon

Department of Plastic and Reconstructive Surgery, Chosun University College of

Medicine, 309 Pilmun-daero, Dong-gu, Gwangju 61452, Korea

E-mail: ps9107@naver.com

*This work was supported by research funds from Chosun University Hospital in 2015.

Received January 19, 2018 / Revised March 9, 2018 / Accepted March 16, 2018 potential legal implications, explanation about the risks of surgery plays an important role in building a trusting relationship between the patient and surgeon, and if such trust is not present, patients can experience discomfort and anxiety, eventually leading to poor satisfaction [2].

Previous reports have stated that patients experience difficulty in remembering verbally delivered information, irrespective of the surgical and medical procedures involved [3,4]. Currently, most informed consent processes involve verbal and written delivery of information [5]. Furthermore, the effects of written information, such as information provided on a paper, in the in- 
formed consent process have been studied in relation to various diseases, and these effects were found to vary widely according to the disease involved [6]. Owing to advances in information technology (IT) and the growing number of smartphone users, mobile applications have recently become a good information delivery tool in terms of portability and accessibility [7]. In this context, we considered effective information delivery using a mobile application in the informed consent process. No previous study has examined the use of a mobile application in the informed consent process for patients with a nasal bone fracture, which is a common condition encountered in the practice of plastic surgery. Most patients with a nasal bone fracture undergo closed reduction surgery [8]. Because its risks and complications are well established [8], an understanding of the surgical risks during the informed consent process may be important.

The purpose of this study was to use a mobile application developed by us in the traditional informed consent process for patients with a nasal bone fracture in order to investigate whether the additional use of our mobile application is more effective than the traditional verbal and written approaches for delivering information, such as surgical risks.

\section{METHODS}

\section{Study design}

This prospective, randomized, single-blind study included 60 patients who underwent nasal bone fracture reduction surgery performed by three plastic surgeons (J.S.C., K.M.S., W.Y.C.) at Chosun University hospital. The patient sample size was calculated with reference to another study about recall of surgical risks [1]. Calculation with $\alpha=0.05$, effect size $=0.5$, and power $=0.80$ identified the requirement of 26 participants in each group (GPower 3.1.9.2; Heinrich Heine University Düsseldorf, Düsseldorf, Germany). Considering potential drop-out, 30 participants were required in the experimental group and 30 in the control group. The inclusion criteria were age 19-65 years, closed reduction surgery for only nasal bone fracture (not combined with other facial bone fractures), and use of an android smartphone. The exclusion criteria were other procedures performed at the same time, inability to communicate in Korean, and unstable general condition. The flow chart for patient assignment and the timeline is presented in Fig. 1. As shown in Fig. 1, the number of recalled surgical risks was assessed using an open question at the

\section{Randomized}

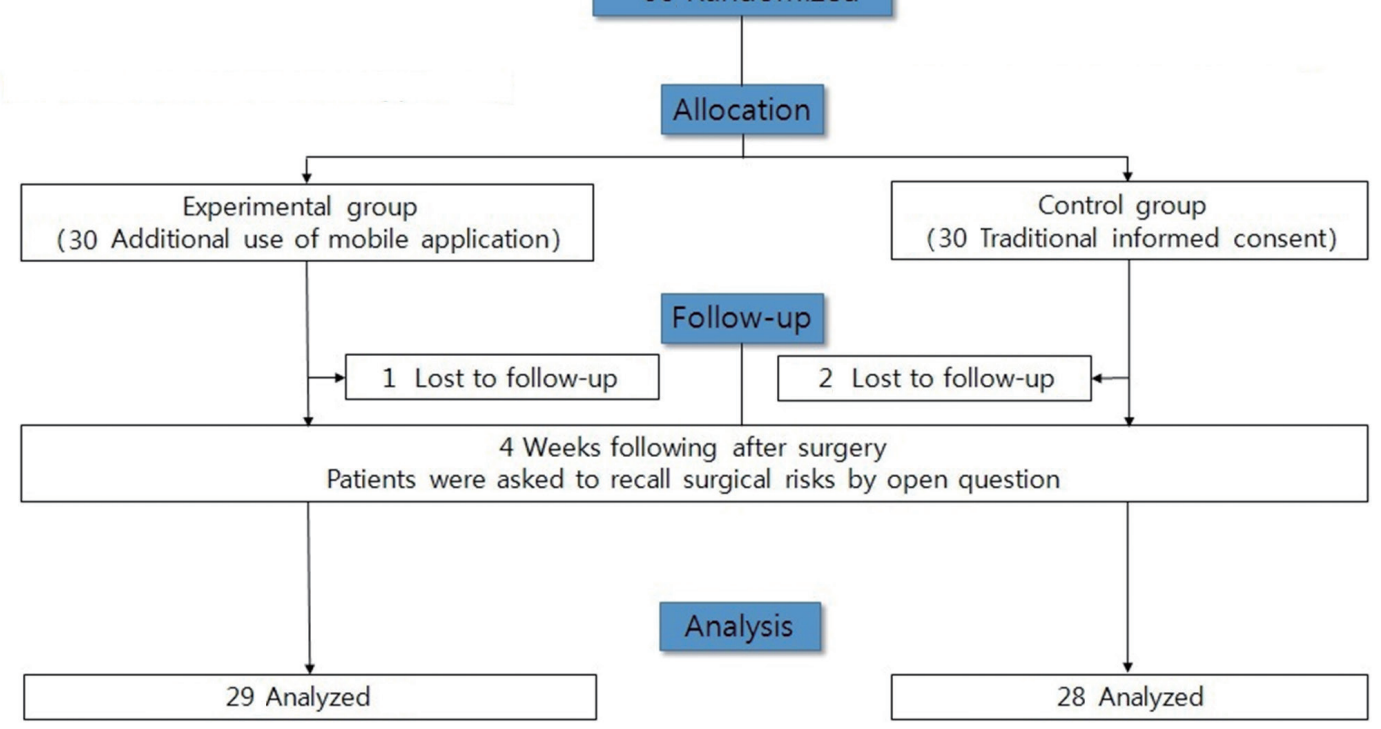

Fig. 1. Flow diagram of study design and patient enrollment methods. 
4-week follow-up visit after surgery. Patient data, including age, sex, and level of education, were collected. The study was approved by Institutional Review Board of Chosun University hospital (IRB No. 2017-06-008-02).

\section{Randomization}

Natural numbers (n) from 1 to 60 were generated using a random number generator. For the "n" of the first 30 numbers that were generated, the "n"th patient was assigned to the experimental group after the start of the study. Additionally, for the " $n$ " of the subsequent 30 numbers, the " $n$ "th patient was assigned to the control group.

\section{Informed consent process and mobile application}

During the informed consent process, patients were explained about their current state, the surgical techniques, and the possible postoperative outcomes and risks [1]. The number of recalled surgical risks after surgery can be used as a parameter to determine how well the patient understood the informed consent process $[1,6,9]$. The control group underwent the traditional informed consent process with verbal descriptions and paper permission. The experimental group used a mobile application developed by our team and underwent the traditional informed consent process with verbal descriptions and paper permission. The participants were able to install the mobile application using a QR code (quick response code)

90101100500003016

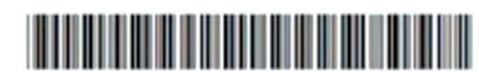

\begin{tabular}{|c|c|c|}
\hline Serial number of patient & & \multirow{4}{*}{$\begin{array}{l}\text { Facial bone fracture treatment } \\
\text { consent and instructions }\end{array}$} \\
\hline Patient name & & \\
\hline Sex/Age & & \\
\hline Department & Room & \\
\hline (Operator): & & (Assistant2) Name: \\
\hline
\end{tabular}

1. Impression :

Your present state : Facial bone fracture (Nasal bone fracture)

Symptom of facial bone fracture : Facial laceration, pain, nasal deformity (depression or deviation), bleeding, obstruction, bruise, numbness, swelling

$\begin{array}{lll}\text { Past history } & \text { Yes / No / Unknown - Allergy } & \text { Yes / No / Unknown } \\ \text { Unusual condition } & \text { Yes / No / Unknown . Diabetes } & \text { Yes / No / Unknown } \\ \text { Hyper/hypotension } & \text { Yes / No / Unknown . Bleeding tendency } & \text { Yes / No / Unknown } \\ \text { Heart disease } & \text { Yes / No / Unknown . Drug accident } & \text { Yes / No / Unknown }\end{array}$

Problem with medicine Yes / No / Unknown

2. Treatment (Operation name)

Operation name: Closed reduction of nasal bone

3. Purpose, need and method of the proposed surgery,

Patient compliance.

A. Purpose

Recovery of facial form

B. Method and materials

(1) Nasal bone fracture : Closed reduction and

Correction of nasal septum
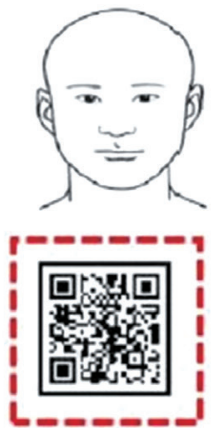

Fig. 2. Inserted QR code (quick response code, red dotted line) for installation of mobile application on the paper permission. 
provided on the paper permission (Fig. 2). The participants were able to enter the operation date; view descriptions about the surgical technique, surgical risks, and postoperative management; and receive notifications through the mobile application (Fig. 3, Supplemental Program File S1.). The following six surgical risks were explained: pain, bleeding, nasal deformity, numbness, nasal obstruction, and nasal cartilage necrosis. The same information was provided on paper and on the mobile application.

\section{Statistical analysis}

Frequency analysis was performed to analyze the total mean number of recalled risks. With regard to the significance of the demographic data in each group classification, sex was analyzed using the independent $t$-test, while age and the level of education were analyzed using the chi-square test. The associations of the number of recalled surgical risks with mobile application use and with sex were analyzed using the independent $t$-test, and the associations with age and the level of education were analyzed using the chi-square test. These analyses were performed using IBM SPSS ver. 19.0 (IBM Corp., Armonk, NY, USA). A p-value $<0.05$ was considered significant.
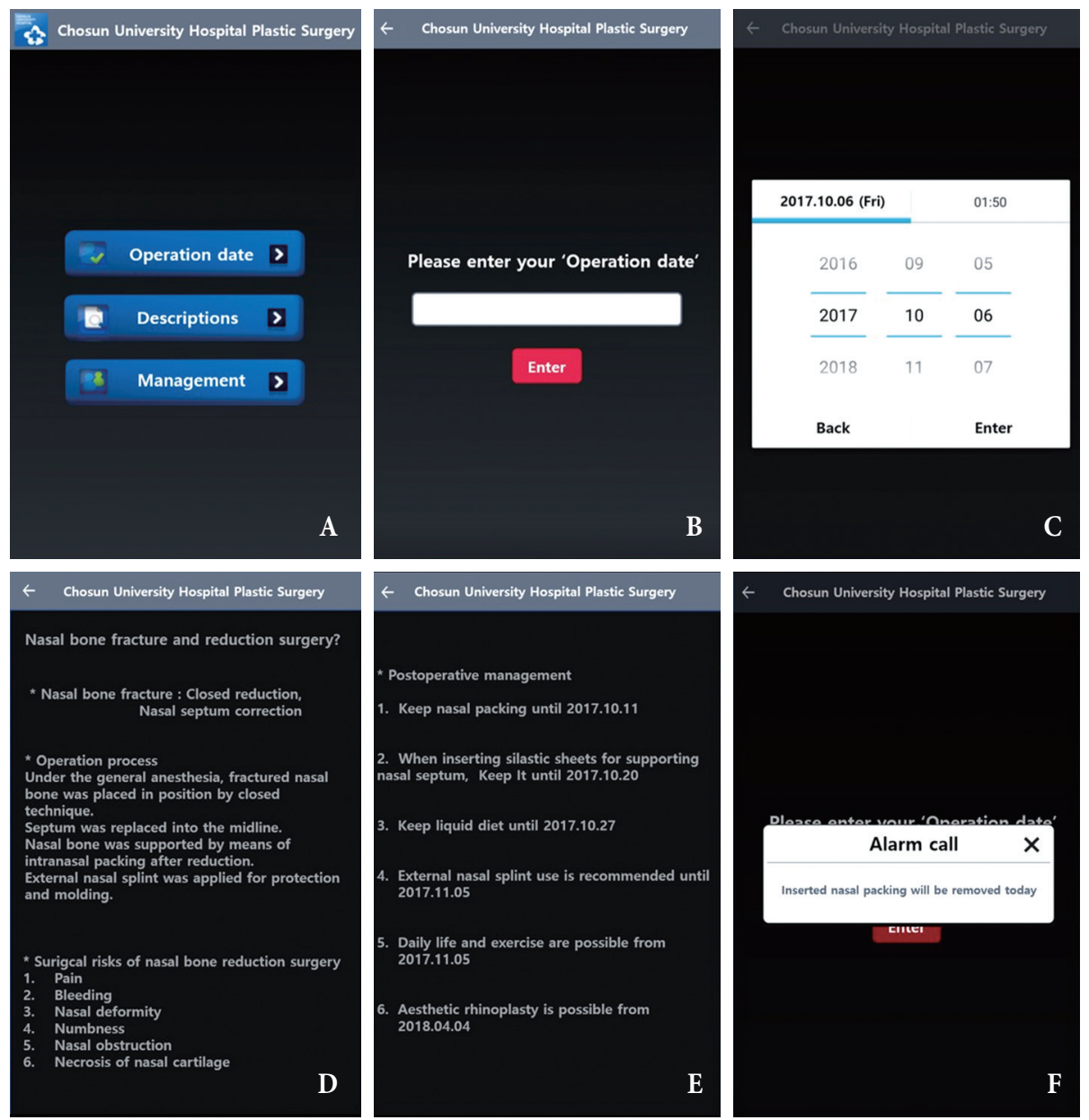

Fig. 3. Screen shots of captured mobile application. (A) Main menu, (B, C) entering the operation date, (D) descriptions about the surgical technique and surgical risks, (E) descriptions about postoperative management, (F) alarm function (notifications through the mobile application). 
Table 1. Baseline characteristics of participants

\begin{tabular}{lccc} 
Variable & $\begin{array}{c}\text { Mobile } \\
\text { application }\end{array}$ & Control & $p$-value \\
Number & 29 & 28 & - \\
Sex & & & 0.430 \\
$\quad$ Male & $20(68.9)$ & $22(78.6)$ & \\
Female & $9(31.1)$ & $6(21.4)$ & \\
Age (yr) & & & 0.247 \\
19-29 & $11(37.9)$ & $10(35.7)$ & \\
$30-39$ & $7(24.1)$ & $8(28.6)$ & \\
$40-49$ & $6(20.7)$ & $8(28.6)$ & \\
$50-59$ & $4(13.7)$ & $2(7.1)$ & \\
$\geq 60$ & $1(3.4)$ & 0 & \\
Level of education & & & 0.171 \\
Less than middle school & $1(3.4)$ & $2(7.1)$ & \\
High school & $11(37.9)$ & $9(32.1)$ & \\
College/university & $17(58.6)$ & $17(60.7)$ & \\
\hline
\end{tabular}

Values are presented as number (\%).

\section{RESULTS}

\section{Demographics}

In total, 30 patients were recruited in the experimental group and another 30 were recruited in the control group. However, three of the 60 recruited patients were lost to follow up (one from the experimental group and two from the control group). The remaining patients were included in the analysis. There were no significant differences in age $(p=0.247)$, sex $(p=0.430)$, and level of education $(p=0.171)$ between the experimental and control groups (Table 1).

\section{Recalled surgical risks}

The mean number of recalled surgical risks among all patients was $1.58 \pm 0.56$. The most frequently recalled surgical risk was nasal deformity in both the experimental and control groups (Fig. 4). The mean number of recalled surgical risks was $1.72 \pm 0.52$ in the experimental group and $1.49 \pm 0.57$ in the control group. There was a significant relationship between the use of the mobile application and the mean number of recalled surgical risks ( $p=0.047$ ). However, the number of recalled surgical risks was not significantly associated with sex $(p=0.399)$, age $(p=0.416)$, and the level of education $(p=0.890)$.

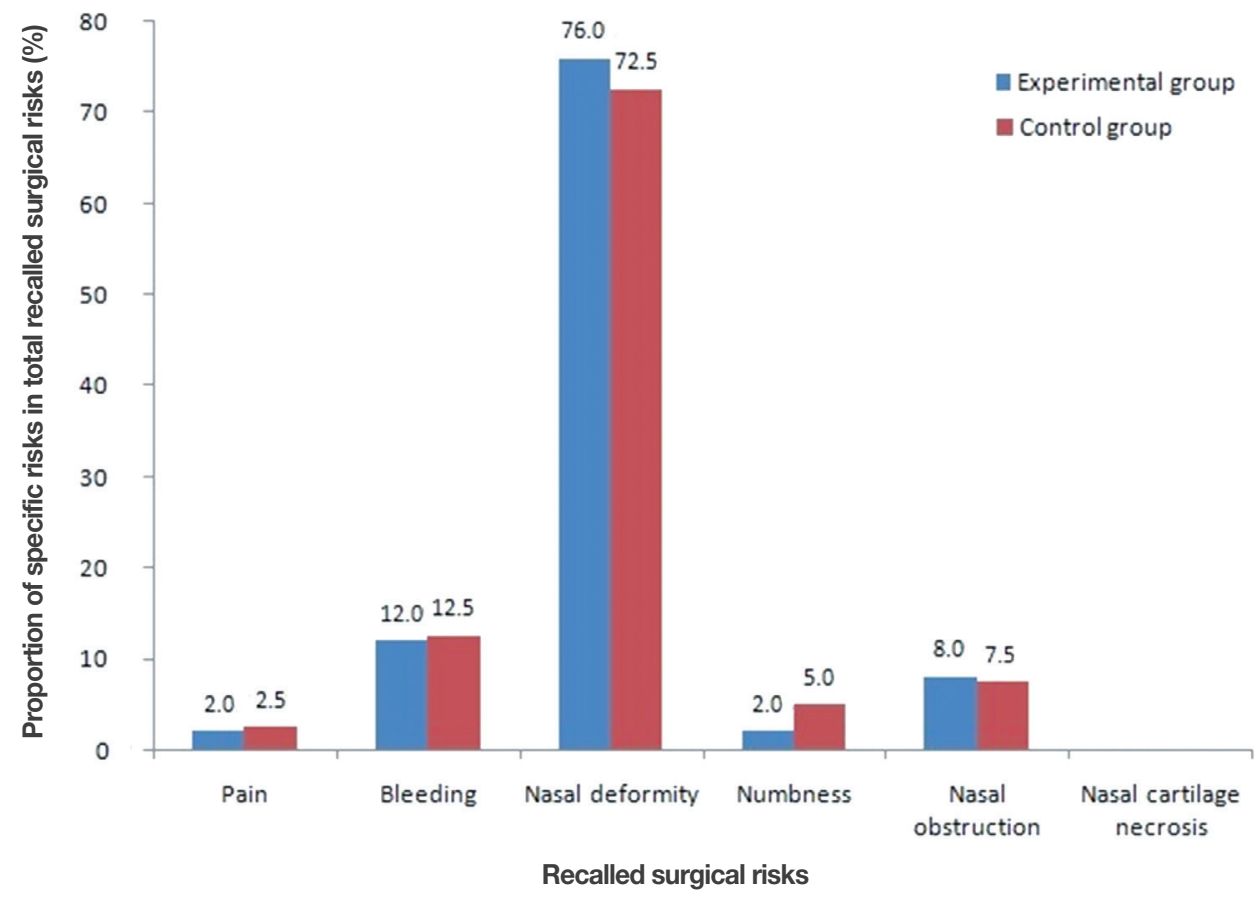

Fig. 4. Percentage of patients who recalled specific risks. 


\section{DISCUSSION}

The informed consent process is an ethically and legally critical process, as it informs patients about their treatment and the relevant processes that they will undergo before and after treatment $[1,2]$. The importance of the informed consent process is further highlighted in cases involving clear risks, potential complications, and subjective assessments of postoperative outcomes. This is because patient satisfaction is not solely dependent on the main procedure but is affected by the overall treatment process, including preoperative explanation and postoperative management [1]. Studies that attempted to improve the effectiveness of information delivery through the traditional verbal and written informed consent process generally educated patients with additional pamphlets and audio-visual presentations, and their efficacy was controversial $[1,10]$.

The rapid advancement of IT and the explosive increase in smartphone access over the last few years have led to the development of IT-based health management systems and devices [7]. Mobile applications are being studied in various medical fields, and they have been suggested to be useful in several aspects, such as delivering medical information and monitoring a patient's sta- tus [7,11]. One notable benefit of delivering explanations about surgical risks through a mobile application during the informed consent process of a surgery, where accurate information delivery and recall are important, is that mobile applications are easily portable and accessible.

This study included patients with a nasal bone fracture, which has clear risks. The mean number of recalled surgical risks for the entire patient group was $1.58 \pm 0.56$, suggesting that most patients remembered only one or two of the six risks explained (Fig. 5). Similarly, the mean numbers of recalled surgical risks in other classic studies were 1.33 and 1.45 when at least five risks were explained initially $[1]$. These results suggest that patients tend to remember the things that are most impactful, possible, and important to them for longer periods when they are explained about several things. The fact that the patients recalled nasal deformity the most reflects the growing interest in beauty and appearance regardless of age and sex and the fundamental purpose of nasal bone fracture reduction.

The higher mean number of recalled surgical risks with the use of a mobile application and traditional approaches that with traditional approaches alone suggests that mobile applications can increase the efficiency of information delivery when compared with

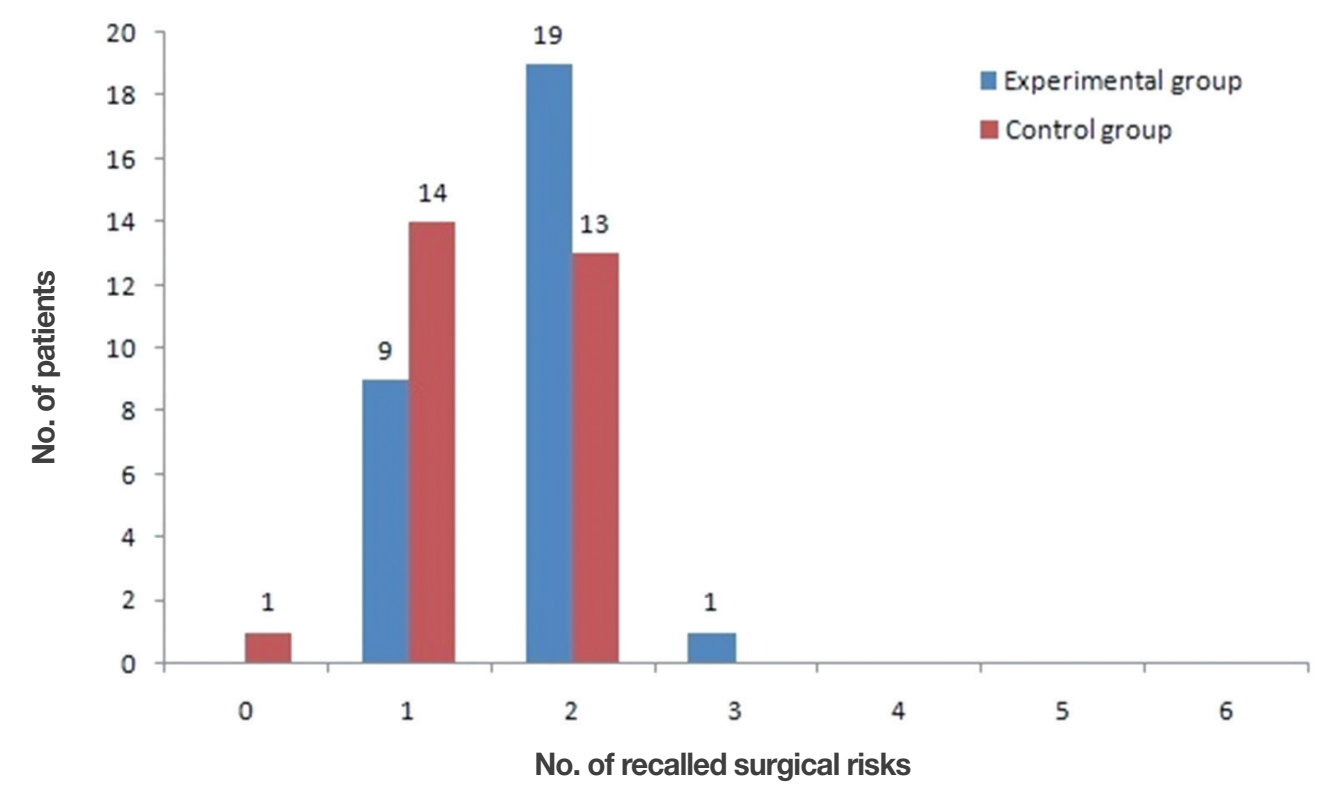

Fig. 5. The number of recalled surgical risks in experimental and control groups. 
other platforms. As previously mentioned, mobile applications can be easily accessed anywhere and at any time, which allows frequent access to information as opposed to one-time access with verbal explanations. Furthermore, the accuracy of information provided by the mobile application was ensured, as it was developed by the healthcare institution and was approved by the IRB, while information viewed on the internet could be inaccurate.

One limitation of this study is that we could not analyze the frequency of the use of the mobile application because this was a blinded study and most patients in the experimental group could not remember how many times they used the mobile application.

In conclusion, this study found that the use of a mobile application contributes to efficient delivery of information in the informed consent process for nasal bone fracture reduction surgery. With further improvement, mobile applications have the potential to be used for other plastic surgeries and other surgeries and could serve as a useful tool for delivery of other types of information in addition to the description of risks.

\section{CONFLICT OF INTEREST}

No potential conflict of interest relevant to this article was reported.

\section{PATIENT CONSENT}

The patients provided written informed consent for the publication and the use of their images.

\section{REFERENCES}

1. Wong AL, Martin J, Tang D, LeBlanc M, Morris SF, Paletz J, et al. The effect of written information on recall of surgical risks of carpal tunnel release surgery: a randomized controlled study. Plast Reconstr Surg 2016;138:1011e-1018e.

2. Langewitz W, Ackermann S, Heierle A, Hertwig R, Ghanim L, Bingisser R. Improving patient recall of information: Harnessing the power of structure. Patient Educ Couns 2015;98:716-21.

3. Kinnersley P, Phillips K, Savage K, Kelly MJ, Farrell E, Morgan B, et al. Interventions to promote informed consent for patients undergoing surgical and other invasive healthcare procedures. Cochrane Database Syst Rev 2013;(7):CD009445.

4. Gillies A, Gillies R, Weinberg L. Patient recollections of perioperative anaesthesia risks. Anaesth Intensive Care 2013;41:247-50.

5. Makdessian AS, Ellis DA, Irish JC. Informed consent in facial plastic surgery: effectiveness of a simple educational intervention. Arch Facial Plast Surg 2004;6:26-30.

6. Hong P, Makdessian AS, Ellis DA, Taylor SM. Informed consent in rhinoplasty: prospective randomized study of risk recall in patients who are given written disclosure of risks versus traditional oral discussion groups. J Otolaryngol Head Neck Surg 2009;38:369-74.

7. Dubey D, Amritphale A, Sawhney A, Amritphale N, Dubey P, Pandey A. Smart phone applications as a source of information on stroke. J Stroke 2014;16:86-90

8. Kang CM, Han DG. Correlation between operation result and patient satisfaction of nasal bone fracture. Arch Craniofac Surg 2017;18:25-9.

9. Nadeau DP, Rich JN, Brietzke SE. Informed consent in pediatric surgery: do parents understand the risks? Arch Otolaryngol Head Neck Surg 2010;136:265-9.

10. Synnot A, Ryan R, Prictor M, Fetherstonhaugh D, Parker B. Audio-visual presentation of information for informed consent for participation in clinical trials. Cochrane Database Syst Rev 2014;(5):CD003717.

11. Kim HS, Choi W, Baek EK, Kim YA, Yang SJ, Choi IY, et al. Efficacy of the smartphone-based glucose management application stratified by user satisfaction. Diabetes Metab J 2014;38:204-10.

Supplemental Program File S1. Mobile application program for android smart phone.

Supplemental data can be found at: https://doi.org/10.7181/acfs.2018.19.1.41 